\title{
Ultrafast Photoinduced Electron Transfer across Semiconductor-Liquid Interfaces in the Presence of Electric Fields
}

\author{
Y. Rosenwaks, ${ }^{*}$ B. R. Thacker, and A. J. Nozik* \\ National Renewable Energy Laboratory, Golden, Colorado 80401 \\ R. J. Ellingson, K. C. Burr, and C. L. Tang \\ Cornell University, Materials Science Center, Ithaca, New York 14853
}

Received: December 7, 1993; In Final Form: January 21, $1994^{\circ}$

\begin{abstract}
The rates of photoinduced electron transfer from $\mathrm{p}-\mathrm{InP}$ to $\mathrm{Fe}(\mathrm{CN})_{6}{ }^{4 / 3-}$ acceptors in aqueous electrolyte have been determined as a function of the initial potential drop (i.e., band bending $\left(V_{\mathrm{BO}}\right)$ ) in the semiconductor space charge layer using femtosecond luminescence up-conversion techniques. The effects of electric field on electron transfer were separated from the effects of field-enhanced charge separation and surface recombination through a rigorous numerical solution of the coupled continuity and Poisson equations using a Cray supercomputer. A very strong dependence of the electron-transfer velocity $\left(S_{\mathrm{et}}\right)$ on $V_{\mathrm{BO}}$ was found, $S_{\mathrm{et}}$ reached a saturation value of $5 \times 10^{7} \mathrm{~cm} / \mathrm{s}$ when the initial value of $V_{\mathrm{BO}}$ in the dark was $\geq 0.5 \mathrm{eV}$. When the initial value of $V_{\mathrm{BO}}$ was set near zero, $S_{\text {et }}$ was $9 \times 10^{3} \mathrm{~cm} / \mathrm{s}$. Hot electron injection processes appear to play a role in this behavior.
\end{abstract}

The dynamics of electron-transfer (ET) processes is a subject of an enormous amount of current experimental and theoretical work. $^{1-15}$ Most of this work deais with intramolecular and intermolecular ET in donor-acceptor molecules, ${ }^{1-6}$ biologicallyderived photosynthetic structures, $, 1,4,7$ and rigid matrices. ${ }^{8}$ Much less work has been reported ${ }^{9-12}$ on the dynamics of ET across semiconductor-liquid junctions; these systems are promising for applications in solar photoconversion processes. ${ }^{13}$

In most published work on intermolecular and intramolecular ET in homogeneous systems, electric field effects are not important or have not been considered. Recently, a few papers have been published describing the effects of externally-applied electric fields on ET in photosynthetic reaction centers. ${ }^{14}$

For heterogeneous ET across metal electrodes, the applied external potential drops across the Helmholtz layer, and the applied potential only affects the Franck-Condon factors for ET through the terms that incorporate the thermodynamic driving force for the reaction, $\Delta G^{\circ}\left(\Delta G^{\circ}=\mathrm{E}-\mathrm{E}^{\circ}\right.$, where $E$ is the electrode potential and $E^{\circ}$ is the formal potential of the redox reaction)..$^{4,15}$

At semiconductor-liquid interfaces, very large electric fields (typically $10^{5}-10^{6} \mathrm{~V} / \mathrm{cm}$ ) exist in the semiconductor close to its surface because of the formation of a space charge layer (SCL). ${ }^{13}$ The potential drop in the SCL produces band bending $\left(V_{B}\right)$. Externally-applied potentials will drop across the SCL (to an extent dependent upon the degree of surface state charging and band-edge movement; see below) to increase or decrease $V_{\mathrm{B}}$. These space charge fields (SCFs) are responsible for the extremely efficient photoinduced charge separation (approaching $100 \%$ quantum yield) that is characteristic of semiconductor-liquid interfaces in photoelectrochemical (PEC) cells. Under bandgap illumination the SCFs are reduced because of screening by the photogenerated majority carriers, but strong residual SCFs are ubiquitous in any operational photoelectrochemical (PEC) cell. The existence of these SCFs is a major difference between ET processes at metal electrodes compared to semiconductor electrodes.

Photoluminescence (PL) experiments designed to measure the rate of ET from semiconductors to redox acceptors in an adjacent solution are usually done with very high-intensity laser excitation in order to flatten the semiconductor bands and reduce the SCF to near zero. The same is done in PL experiments designed to

- Abstract published in Advance ACS Abstracts, March 1, 1994. measure the rate of surface recombination in illuminated semiconductors. ${ }^{16,17}$ This is because the presence of electric field terms greatly complicates the solution of the coupled continuity and Poisson equations that is needed to relate the observed rate of photogenerated carrier decay to the rate of surface recombination or electron transfer.

In the present work we have rigorously solved the continuity and Poisson equations using a finite-difference semiimplicit algorithm with a Cray-2 supercomputer and have applied the results to analyze time-resolved PL decay experiments in the presence of large SCFs that are controlled with an externally applied bias voltage. Our model calculations allow us to separate the effects on PL quenching of field-enhanced ET from the effects of simple field-enhanced charge separation and surface recombination. ${ }^{18,19}$ The photoelectrode is p-InP in contact with the redox couple $\mathrm{Fe}(\mathrm{CN})_{6}{ }^{3-/ 4}$ in aqueous electrolyte; the PL decay dynamics were measured using PL up-conversion techniques ${ }^{20,21}$ with a time resolution of $100 \mathrm{fs}$. Our results show increasing and surprisingly high ET rates (electron-transfer velocities $>10^{7} \mathrm{~cm} /$ s) as the applied potential is increased. The significance of these results for models of photoinduced ET at semiconductor-liquid junctions is discussed below.

The InP samples studied were p-type ( $\mathrm{Zn}$ doped, $1.5 \times 10^{17}$ $\mathrm{cm}^{-3}$ ) single crystals with (100) surface orientation (from Nippon Mining). They were etched for $30 \mathrm{~s}$ in $2 \% \mathrm{Br}_{2} / \mathrm{MeOH}$ and then mounted in a sealed oxygen-free PEC cell. The electrolyte used was sodium sulfate; all the potentials were measured relative to a sodium-saturated reference electrode (SSCE).

Time-resolved PL (TRPL) spectra were obtained by the technique of sum frequency generation. ${ }^{20,22}$ Photoexcitation was with a Ti:sapphire laser that provided 80 -fs pulses at a wavelength of $780 \mathrm{~nm}$ and $82-\mathrm{MHz}$ repetition rate. The laser spot size was $\approx 20 \mu \mathrm{m}$ in diameter, and the temporal resolution of the system was $100 \mathrm{fs}$. The band-edge TRPL was measured at different potentials applied to the InP photoelectrode. The potentials were controlled with a potentiostat; Mott-Schottky (MS) and current vs voltage $(i-V)$ data were also obtained using the Ti:sapphire excitation beam. All the MS measurements were conducted at a frequency of $20 \mathrm{kHz}$.

The experiments were conducted as follows: (a) first, the TRPL of the etched electrode under open circuit conditions (OCTRPL) immersed in the electrolyte was recorded in order to determine 


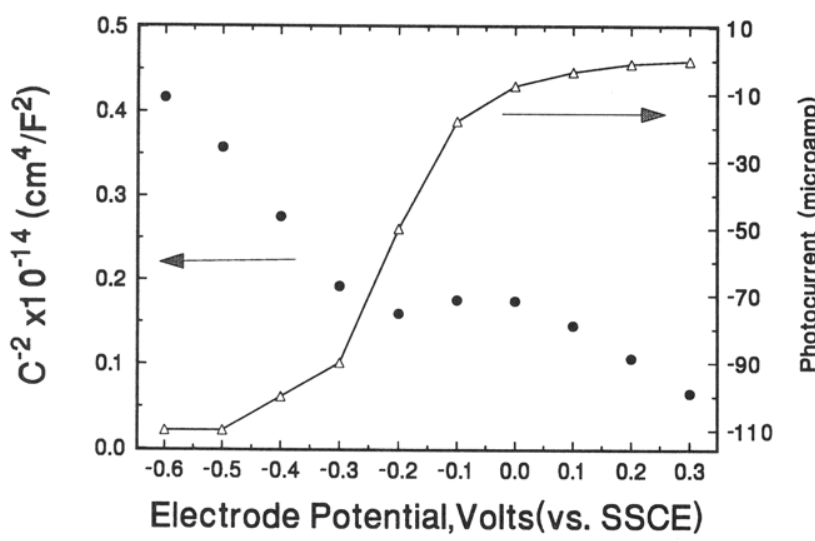

Figure 1. Mott-Schottky plot of $\mathrm{p}$-InP sample in $0.1 \mathrm{M} \mathrm{Fe}(\mathrm{CN})_{6}{ }^{4-3-}$ under laser excitation superimposed on photocurrent-potential plot.

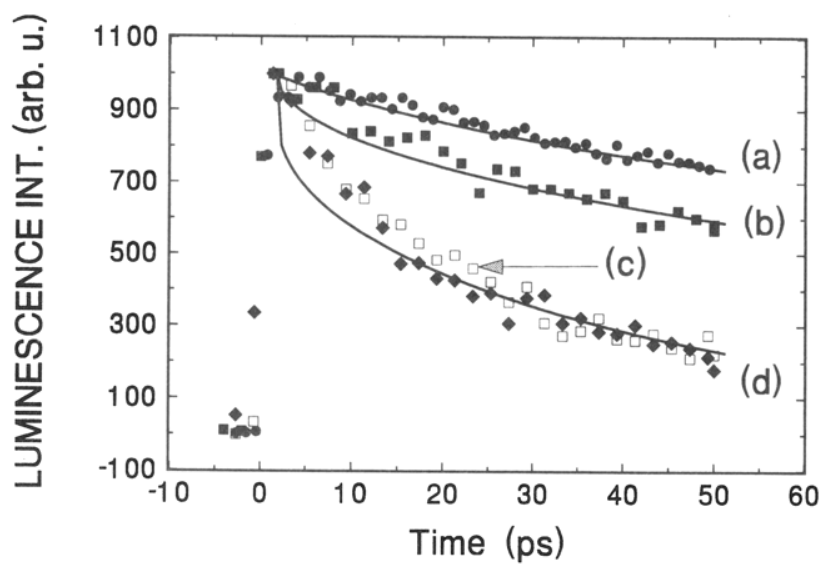

Figure 2. PL decay data and its fit with model for p-InP in $0.1 \mathrm{M}$ $\mathrm{Fe}(\mathrm{CN})_{6}{ }^{4-3-}$ at four electrode potentials: (a) $E=+0.3 \mathrm{~V}, V_{\mathrm{BO}}=0.07$ $\mathrm{eV}$; (b) $E=-0.3 \mathrm{~V}, V_{\mathrm{BO}}=0.37 \mathrm{eV}$; (c) $E=-0.5 \mathrm{~V}, V_{\mathrm{BO}}=0.57 \mathrm{eV}$; (d) $E=-1.0 \mathrm{~V}, V_{\mathrm{BO}}=1.07 \mathrm{eV}$.

the crystal surface recombination velocity (SRV); (b) then the acceptor was added to the cell and the OCTRPL remeasured in order to verify that the SRV was unchanged in zero field as a result of the presence of the acceptor molecules; (c) the MS and the $i-V$ were then measured (both in the dark and under the same laser-illumination conditions used in the TRPL experiments) in order to extract the flatband potential $\left(V_{\mathrm{fb}}\right)$ and $V_{\mathrm{B}}$; (d) then the OCTRPL was remeasured in order to verify that the electrode SRV had not changed during the measurements in (c); (e) the TRPL was then measured at different applied biases to vary $V_{\mathrm{B}}$ from 0 to $>1 \mathrm{eV}$; (f) finally, stages (b) and (c) were repeated in order to check whether any permanent photochemical changes had occurred on the InP surface during stage (e).

Figure 1 shows the photo $i-V$ and the MS plot of the InP electrode in $0.1 \mathrm{M} \mathrm{Fe}(\mathrm{CN})_{6}^{3-/ 4-}$ measured under the laser illumination. In the dark the MS plot is linear from $V_{\mathrm{fb}}(0.39$ $\mathrm{V})$ to $-1.0 \mathrm{~V}$. Under illumination the MS plot is linear from the dark $V_{\mathrm{fb}}(0.37 \mathrm{~V})$ to about $0.05 \mathrm{~V}$; it becomes flat between 0.05 and $-0.25 \mathrm{~V}$ and becomes linear again at potentials negative of about $-0.25 \mathrm{~V}$. The flat region in the MS plot indicates negative surface state charging and negative band-edge movement; ${ }^{23 a}$ the value of $V_{\mathrm{fb}}$ after band movement in the light is completed is $0.0 \mathrm{~V}$. $V_{\mathrm{B}}$ changes linearly with the applied potential in the two linear regions of the MS plot. As the surface state charging process begins (at $\approx 0.0 \mathrm{~V}$ ), the photocurrent increases and saturates at a potential of about $-0.5 \mathrm{~V}$.

Figure 2 shows the TRPL decay of the InP electrode in $0.1 \mathrm{M}$ $\mathrm{Fe}(\mathrm{CN})_{6}{ }^{3-/ 4}$ measured at four different electrode potentials: 0.3 , $-0.3,-0.5$, and $-1.0 \mathrm{~V}$. The solid lines were calculated from our model as outlined briefly below. Using our model, we can calculate the electron-transfer velocity, $S_{\mathrm{et}}\left(S_{\mathrm{et}}=k_{\mathrm{et}} C_{\mathrm{acc}}\right.$, where $k_{\mathrm{et}}$ is the second-order rate constant for ET and $C_{\text {acc }}$ is the acceptor

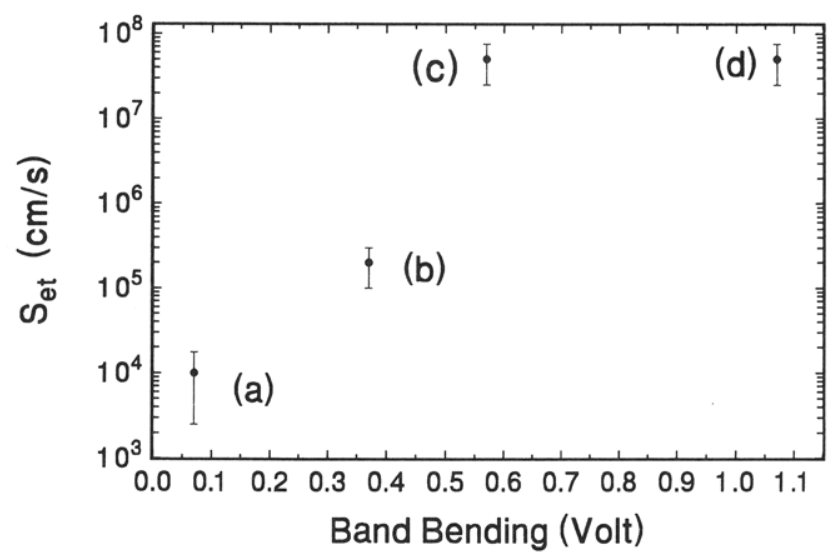

Figure 3. Plot of electron-transfer velocity $\left(S_{\mathrm{et}}\right)$ vs initial band bending in the dark $\left(V_{\mathrm{BO}}\right)$.

concentration), from the PL decay data. Figure 3 shows $S_{\text {et }}$ plotted as a function of the initial band bending in the dark $\left(V_{\mathrm{BO}}\right)$. The band bending is calculated from $V_{\mathrm{BO}}=E-V_{\mathrm{fb}}$, where band movement is taken into account.

Figure 3 shows clearly that $S_{\text {et }}$ has a very strong dependence on $V_{\mathrm{BO}} . S_{\text {et }}$ increases from $9 \times 10^{3} \mathrm{~cm} / \mathrm{s}$ with $V_{\mathrm{BO}}=0.07 \mathrm{eV}$ to $\approx 5 \times 10^{7} \mathrm{~cm} / \mathrm{s}$ with $V_{\mathrm{BO}} \geq 0.5 \mathrm{eV} ; S_{\mathrm{et}}$ saturates when $V_{\mathrm{BO}}>0.5$ $\mathrm{eV}$. Comparing Figure 3 with Figure 1 reveals that the most rapid rise in $S_{\text {et }}$ occurs after surface state charging and bandedge movement have been completed and that the saturation of $S_{\text {et }}$ and the photocurrent occurs at the same $V_{\text {BO }}$ of $0.5 \mathrm{eV}$.

The TRPL was also measured as a function of $V_{\mathrm{BO}}$ without a redox acceptor present in the solution; this control experiment showed a much smaller decay rate compared to the cases with the acceptor present. Also, we found that some acceptors showed relatively slow ET that did not improve with increased $V_{\text {BO }}{ }^{19}$ These results further support our conclusion that the PL quenching we observe with $\mathrm{Fe}(\mathrm{CN})_{6}{ }^{3-/ 4}$ is caused by ET and not by some other field-quenching mechanism.

The procedure used for modeling the TRPL spectra in the presence of electric fields is now briefly described; a full description will be published elsewhere. ${ }^{18,19}$ It is based on a numerical solution of the continuity equations for electrons and holes coupled through the electric field which is calculated via Poisson's equation in a self-consistent way. The model takes into account (a) electron and hole surface recombination, (b) electron and hole transfer to the adjacent media (i.e., to the solution at the front surface and to the ohmic contact at the back), and (c) radiative and nonradiative bulk recombination throughout the semiconductor.

The calculations of the theoretical PL decay curves (solid lines) in Figure 2 were done as follows. First, a fit to the OCTRPL data (top curve) was obtained. The following known parameters were used: $\alpha$ (absorption coefficient at the exciting laser energy) $=2.5 \times 10^{4} \mathrm{~cm}^{-1}, \alpha_{\mathrm{L}}$ (absorption coefficient at the band gap $)=$ $1 \times 10^{3} \mathrm{~cm}^{-1}, \mu_{\mathrm{n}}$ (electron mobility) $=1900 \mathrm{~cm}^{2} \mathrm{~V}^{-1} \mathrm{~s}^{-1}, \mu_{\mathrm{p}}$ (hole mobility) $=100 \mathrm{~cm}^{2} \mathrm{~V}^{-1} \mathrm{~s}^{-1}, B$ (the second-order radiative rate constant) $=2 \times 10^{-10} \mathrm{~cm}^{3} \mathrm{~s}^{-1}$, and $\tau_{\mathrm{n}}$ (electron bulk nonradiative lifetime $)=\tau_{\mathrm{p}}($ hole bulk nonradiative lifetime $)=0.5 \mathrm{~ns}$; selection of the values of the above fixed parameters was based on a recent study of InP. ${ }^{24} \mathrm{~A} \delta(t)$ laser pulse was assumed, and the injection level, $\mathrm{I}_{0}$ (defined here as the ratio between the initial (at $t=0$ ) excess carrier concentration and the semiconductor doping), was calculated to be 3 based on the measured laser spot size and incident laser power. The values of the two adjustable parameters, $S_{\mathrm{nf}}$ and $S_{\mathrm{pf}}$ (defined as the SRV for electrons and holes at the front liquid interface, respectively), required for the best fit of the data were $S_{\mathrm{nf}}=S_{\mathrm{pf}}=9 \times 10^{3} \mathrm{~cm} / \mathrm{s}$.

The calculation of the bottom two solid lines was done using the same values of the parameters described above, except that $V_{\mathrm{B}}$ at $t<0\left(V_{\mathrm{BO}}\right)$ was fixed at different values depending upon the electrode potential and the band movement, and the adjustable 
parameter $S_{\text {et }}$ was optimized to obtain a good fit. The results were as follows: for curve (b), $V_{\mathrm{BO}}=0.3 \mathrm{eV}$ and $S_{\mathrm{et}}=2 \times 10^{5}$ $\mathrm{cm} / \mathrm{s}$; for curves (c) and (d), $V_{\mathrm{BO}}=0.5$ and $1.0 \mathrm{eV}$, respectively, and $S_{\mathrm{et}}=5 \times 10^{7} \mathrm{~cm} / \mathrm{s}$ for both $V_{\mathrm{BO}}$ values. The uncertainty in the $S_{\text {et }}$ values is shown in Figure 3 by the error bars which represent the $S_{\text {et }}$ range that yields similiar goodness of fits to the experimental data. The relatively poorer fit to the data for curves (c) and (d) at times $<10 \mathrm{ps}$ is attributed to the fact that it takes on the order of $10 \mathrm{ps}$ for the hot electrons created by the excitation pulse to reach the bottom of the conduction band. ${ }^{21,22,25}$ Our model currently does not take hot electron cooling effects into account.

Our results clearly demonstrate that the experimental data must be fitted with a field-dependent electron-transfer velocity, $S_{\text {et }}$. Attempts to fit the experimental data in Figure 2 with a constant $S_{\mathrm{et}}$ of $9 \times 10^{3} \mathrm{~cm} / \mathrm{s}$ and increasing $V_{\text {BO }}$ resulted in decay curves similar to the top one. The possibility that the change in the TRPL spectra with increased reverse bias potential is caused by field-enhanced surface recombination is rejected for three reasons: (1) An increased SRV is inconsistent with the increase in photocurrent seen in Figure 1 as the reverse bias potential is increased. (2) As the applied potential becomes more negative and more surface states are filled as indicated by the flat region in the MS plot in Figure 1, the SRV should decrease. (3) An SRV increase is commonly associated with permanent irreversible surface damage; however, remeasuring the TRPL at the same spot after returning to open circuit conditions gave the identical decay curve as measured previously.

Calculations based on our model show ${ }^{19}$ that, under the high injection conditions in the present experiments $\left(I_{0}=3\right)$, the band bending under illumination is reduced to about $0.1 \mathrm{eV}$ within a picosecond for all values of $V_{\mathrm{BO}}$. Nevertheless, the electric field at the semiconductor surface, $F_{\mathrm{s}}$, remains above $1 \times 10^{5} \mathrm{~V} / \mathrm{cm}$ during the first $100 \mathrm{ps}$; for example, when $V_{\mathrm{BO}}=0.3 \mathrm{eV}, F_{\mathrm{s}}$ is about $1 \times 10^{5} \mathrm{~V} / \mathrm{cm}$, and when $V_{\mathrm{BO}}$ is $0.5 \mathrm{eV}, F$ is about $2.3 \times$ $10^{5} \mathrm{~V} / \mathrm{cm}$. These large $F_{\mathrm{s}}$ values may affect various factors that control the electron-transfer rate. One certain factor is that the fields in the SCL easily create type I hot carriers at the InPliquid interface. ${ }^{21,25}$ Since the transit time of hot electrons across the SCL is extremely fast ( $\approx 100 \mathrm{fs}$ ), the hot electrons arrive at the surface with energies that are determined by the energy level of the field-free conduction band edge in the dark.

The observation of a field-dependent $S_{\mathrm{et}}$ that can reach values above $10^{7} \mathrm{~cm} / \mathrm{s}$ is very interesting; these $S_{\mathrm{et}}$ values correspond to ET times faster than $3 \mathrm{ps}\left(\approx 0.7 \mathrm{ps}\right.$ for $\left.S_{\mathrm{et}}=5 \times 10^{7} \mathrm{~cm} / \mathrm{s}\right)$. There are three possible mechanism by which the ET rate constant can increase with applied electric fields: (a) band-edge movement caused by the applied field could improve the overlap of the conduction band edge with the redox acceptor energy levels (increased Franck-Condon factor); (b) hot electron injection processes induced by the field could also increase the FranckCondon factor because the negative $\Delta G$ for the ET is increased (by as much as $V_{\text {BO }}$ for type I hot carrier injection ${ }^{23}$ ); and (c) field-induced hot electron injection could increase the electronic coupling through enhanced tunneling across the semiconductorto-acceptor barrier and through greater overlap of the wave functions of the injected conduction band electrons with that of the acceptor states.

Figure 3 shows that $S_{\text {et }}$ increases by about 3 orders of magnitude when the electrode potential is changed from 0.3 to $-0.5 \mathrm{~V}$ (accompanied by band-edge movement of about $0.3 \mathrm{eV}$ ). If the maximum value of $S_{\mathrm{et}}$ is taken to coincide with the peak of the Gaussian distribution of acceptor states, then the decrease in the ET rate constant over $0.3 \mathrm{~V}$ (assuming a reorganization energy of $1.0 \mathrm{eV}$ for $\mathrm{Fe}(\mathrm{CN})_{6}^{3-14-27}$ ) would be about a factor of 10 . Hence, mechanism (a) above cannot account for the observed total increase in $S_{\mathrm{et}}$ with increased reverse bias potential; it appears that mechanisms (b) and/or (c) play an important role.
It is interesting to note that the saturation value for $S_{\mathrm{et}}$ is about the same as the saturated drift velocity of electrons in InP;28 this suggests that at high $V_{\mathrm{B}}$ transport in the $\mathrm{InP}$ is the rate-limiting step for ET.

It is clear from calculations ${ }^{19,23 a}$ and other experiments ${ }^{29}$ that in the present experiments hot electrons arrive at the surface with a total energy equal to that in the field-free region of the conduction band and with a kinetic energy equal to $V_{\mathrm{BO}}$. The observed dependence of electron-transfer kinetics on $V_{\mathrm{BO}}$ appears to be correlated with the variation of hot electron energies with $V_{\mathrm{BO}}$. Further studies are in progress to understand the origin of the increase in $S_{\mathrm{et}}$ with electric field quantitatively and to assess in detail the role of hot electron injection and carrier velocity (viz. kinetic energy) on both Franck-Condon and electronic coupling factors.

Acknowledgment. This work was funded by the U.S. Department of Energy, Office of Energy Research, Office of Basic Energy Sciences, Division of Chemical Sciences. R.J.E., K.C.B., and C.L.T. were supported by the National Science Foundation and the Joint Services Electronics Program.

\section{References and Notes}

(1) Fox, M. A., Chanon, M., Eds. Photoinduced Electron Transfer, Parts A-D; Elsevier: Amsterdam, 1988.

(2) Special Issue on Electron Transfer, Chem. Phys. 1993, $176(2,3)$.

(3) Marcus, R. J. Chem. Phys. 1956, 24, 966; Annu. Rev. Phys. Chem. $1964,15,155$

(4) Marcus, R. A.; Sutin, N. Biochim. Biophys. Acta 1985, 811, 265.

(5) Newton, M. D.; Sutin, N. Annu. Rev. Phys. Chem. 1984, 35, 437.

(6) Closs, G. L.; Miller, J. R. Science 1988, 240, 440.

(7) Budil, D. E.; Gast, P.; Chang, C. H.; Schiffer, M.; Norris, J. Annu. Rev. Phys. Chem. 1987, 38, 561.

(8) Miller, J. R.; Beitz, J. V. J. Chem. Phys. 1981, 74, 6476. Huddleston, R. K. J. Am. Chem. Soc. 1984, 106, 5057.

(9) Gomez-Jahn, L. A.; Miller, R. J. D. J. Chem. Phys. 1992, 96, 3981.

(10) Kasinski, J. J.; Gomez-Jahn, L. A.; Faran, J. J.; Gracewski, S. M.; Miller, R. J. D. J. Chem. Phys. 1989, 90, 1253-1268.

(11) Forbes, M. D. E.; Lewis, N. S. J. Am. Chem. Soc. 1990, 112, 3683.

(12) Rosenwaks, Y.; Thacker, B. R.; Ahrenkiel, R. K.; Nozik, A. J. J. Phys. Chem. 1992, 96, 10096.

(13) Nozik, A. J. Annu. Rev. Phys. Chem. 1978, 29, 189.

(14) Lockart, D. J.; Kirmaier, C.; Holten, D.; Boxer, S. G. J. Phys. Chem. 1990, 94, 6987. Franzen, S.; Goldstein, R. F.; Boxer, S. G. J. Phys. Chem. 1990, 94, 5135; 1993, 97, 6304. Gopher, A.; Schonfeld, M.; Feher, G. Biophys. J. 1985, 48, 311-320.

(15) Chidsey, C. E. D. Science 1991, 251, 919.

(16) Ahrenkiel, R. Semiconductors and Semimetals; Academic Press: London, 1993; Vol. 39.

(17) Rosenwaks, Y.; Shapira, Y.; Huppert, D. Phys. Rev. B 1992, 45, 9108

(18) Rosenwaks, Y.; Nozik, A. J.; Yavneh, I. J. Appl. Phys., in press.

(19) Rosenwaks, Y.; Yavneh, I.; Thacker, B.; Nozik, A. J. To be published.

(20) Mahr, H.; Hirsch, M. D. Opt. Commun. 1975, 13, 96.

(21) Pelouch, W. S.; Ellingson, R. J.; Powers, P. E.; Tang, C. L.; Szymd, D.; Nozik, A. J. Phys. Rev. B 1992, 45, 1450; SPIE 1992, 1677, 260.

(22) Wise, F.; Tang, C. L. Solid State Commun. 1989, 69, 821

(23) (a) Copper, G.; Turner, J. A.; Parkinson, B. A.; Nozik, A. J. J. Appl. Phys. 1983, 54, 6463. (b) Koval, C. A.; Torres, R. J. Am. Chem. Soc. 1993, 115,8368 .

(24) Rosenwaks, Y.; Thacker, B. R.; Nozik, A. J.; Shapira, Y.; Huppert, D. J. Phys. Chem. 1993, 97, 10421.

(25) Rosenwaks, Y.; Hanna, M. C.; Levi, D.; Szmyd, D.; Ahrenkiel, R. K.; Nozik, A. J. Phys. Rev. B 1993, 48, 14675.

(26) Zhou, X.; Hsiang, T. Y.; Miller, R. J. D. J. Appl. Phys. 1989, 66, 3066.

(27) Frese, K. W. J. Phys. Chem. 1981, 85, 3911.

(28) Adachi, S. Physical Properties of III-V Semiconductor Compounds; Wiley-Interscience: New York, 1992; p 240.

(29) Min, L.; Miller, R. J. D. Appl. Phys. Lett. 1990, 56, 524. 